\title{
Design and Cytotoxic Evaluation of New Annonaceous Acetogenin Analogues
}

\author{
Jürgen Krauss, * Franz, Bracher, Katrin Synowitz, and Doris Unterreitmeier \\ Department Pharmazie, Zentrum für Pharmaforschung \\ Ludwig-Maximilians, Universität München, Butenandtstr. 5-13, 81377 Munich, Germany
}

\begin{abstract}
Acetogeninas anonáceas análogas foram preparadas a partir de 5-iodofurano-2-carbaldeído e ácido undec-10-inoico ou undec-10-inol pela reação de Sonogashira, seguida da reação de Grignard e hidrólise catalizada por mercúrio. A citocidade foi avaliada por ensaios MTT contra células HL e no Instituto Nacional de Câncer (Alemanha).
\end{abstract}

Analogues of annonaceous acetogenins were built up from 5-iodofuran-2-carbaldehyde and undec-10-ynoic acid or undec-10-ynol by a Sonogashira reaction, followed by a Grignard reaction and a mercury catalysed hydratisation. The cytotoxicity was evaluated with MTT assay ((3(4,5-dimethylthiazol-2-yl)-2,5-diphenyltetrazolium bromide colorimetric assay for measuring cellular proliferation) against HL cells and at the National Cancer Institute (NCI).

Keywords: Sonogashira reaction, Grignard reaction, cytotoxicity

\section{Introduction}

The tropical plant family of the Annonaceae contains pharmacologically active natural products of the group of alkaloids and acetogenins. These plants are often used in traditional medicine. The annonaceous acetogenins are an interesting target for the development of new anti cancer drugs. Compounds like asitrocin exhibit high selectivity against several cancer cell lines. ${ }^{1,2}$ On the other hand, the the total syntheses reported up to know are long and expensive, ${ }^{1,7,8}$ so we tried a simpler approach towards some analogues. The mechanism of action of the annonaceous acetogenins is closed to the cellular mitochondria, following inhibition of the mitochondrial complex I, leading to a lower production of ATP in the tumor cell and subsequent apoptosis.

\section{Results and Discussion}

\section{Chemistry}

In continuation of our work on the synthesis of analogues of annonaceous acetogenin analogues ${ }^{3}$ we describe here a new approach towards the acetogenin skeleton. Commercially available 5-iodofuran-2carbaldehyde (Aldrich) (1) was reacted in a Sonogashira

*e-mail: hjkra@cup.uni-muenchen.de reaction with undec-10-yn-1-ol to give the aldehyde $\mathbf{3}$. This aldehyde $\mathbf{3}$ was converted in a Grignard reaction with dodecyl magnesium bromide to the racemic diol 4. Subsequent regioselective hydratisation ${ }^{4}$ of the triple bond under mercury catalysis led to the corresponding ketone $\mathbf{5}$.

In a second series, undec-10-ynoic acid was esterified via the acid chloride with methanol to give the methyl ester $\mathbf{2 a}$ and with lactic acid ethyl ester to give the double ester $\mathbf{2 b}$. These esters $\mathbf{2 a}$ and $\mathbf{2 b}$ were reacted with 5-iodofuran-2-carbaldehyde (1) under Sonogashira conditions to give the esters $\mathbf{6 a}$ and $\mathbf{6 b}$. In a Grignard reaction with one equivalent of dodecylmagnesium bromide $\mathbf{6 a}$ and $\mathbf{6} \mathbf{b}$ were converted to compounds $\mathbf{7 a}$ and $\mathbf{7 b}$.

\section{Antimicrobial and cytotoxicity screening}

The resulting compounds were tested against several bacteria and fungi in an agar diffusion assay, but did not show any significant activities.

The cytotoxicity of $\mathbf{3}, \mathbf{4}, \mathbf{6 b}$ and $\mathbf{7 b}$ was evaluated in MTT assay against a HL 60 cell line using the method of Mosman. ${ }^{5}$ The results were compared to common alkylating drug cisplatin. Compounds $\mathbf{4}$ and $\mathbf{7 a}$ were also tested at the NCI against 60 cancer cell lines, but showed only weak cytotoxicity and no cellular selectivity. ${ }^{6}$ 


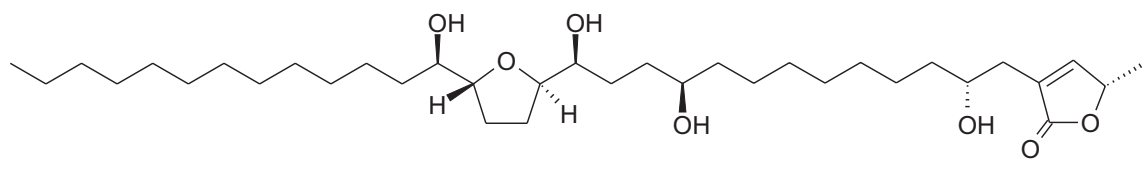

asitrocin

Scheme 1. Annonaceae acetogenin asitrocin.

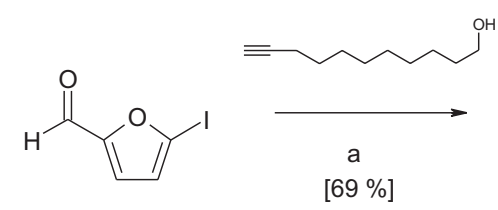<smiles>O=Cc1ccc(C#CCCCCCCCCCO)o1</smiles>

3
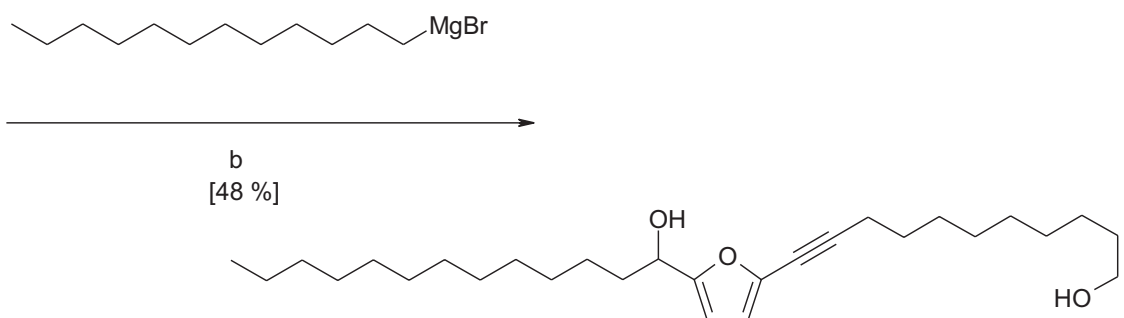

4

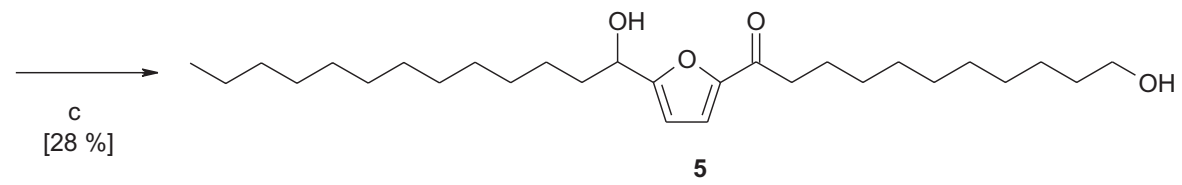

Scheme 2. a: EDMA, $\mathrm{Pd}\left(\left(\mathrm{PPh}_{3}\right)_{2}\right) \mathrm{Cl}_{2}$, CuI; b: THF; c: $\mathrm{HgO}, \mathrm{H}_{2} \mathrm{O}, \mathrm{HOAc}$.
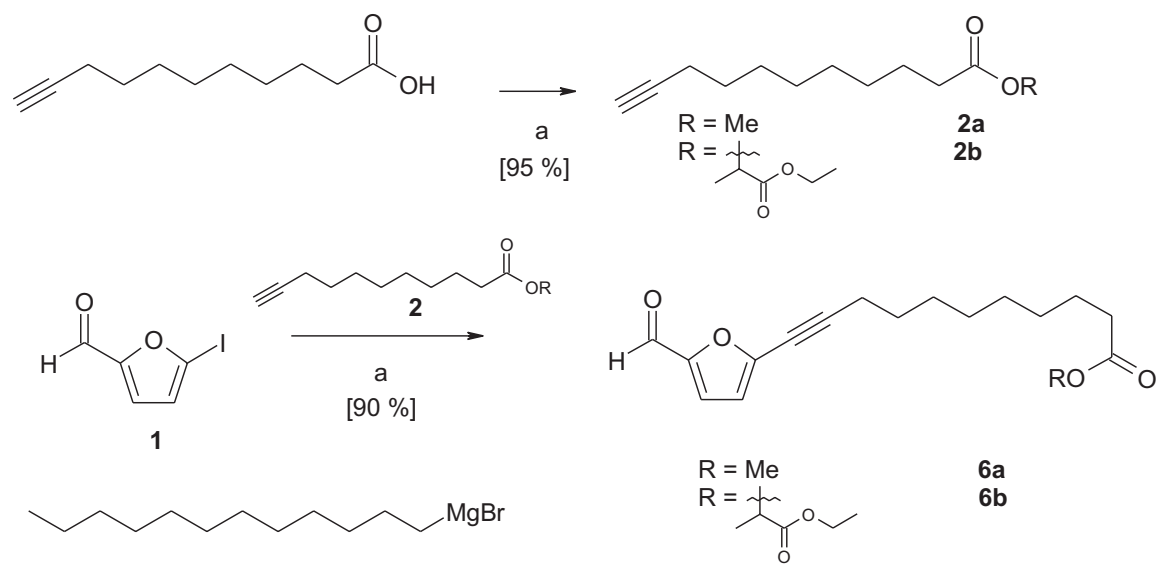

$6 a$

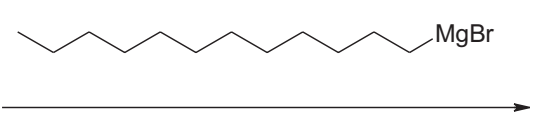

c

[62-78\%]

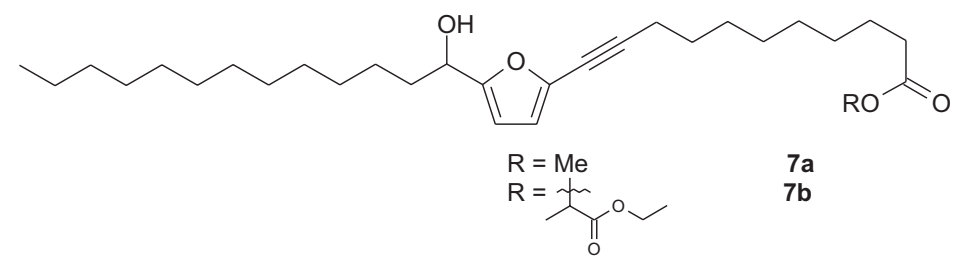

Scheme 3. a: $\mathrm{C}_{2} \mathrm{Cl}_{2} \mathrm{O}_{2}$, EDMA, methanol. b: EDMA, $\mathrm{Pd}\left(\left(\mathrm{PPh}_{3}\right)_{2}\right) \mathrm{Cl}_{2}$, CuI. c: THF. 
Table 1. Cytotoxicity aganist HL 60 cell line determined with MTT test

\begin{tabular}{lccc}
\hline compound & $\mathrm{IC}_{50} / \mu \mathrm{M}$ & compound & $\mathrm{IC}_{50} / \mu \mathrm{M}$ \\
\hline $\mathbf{2 a}$ & 1000 & $\mathbf{2 b}$ & 4000 \\
$\mathbf{3}$ & 30 & $\mathbf{6 a}$ & 400 \\
$\mathbf{4}$ & 30 & $\mathbf{6 b}$ & 2000 \\
cisplatin & 5 & $\mathbf{7 b}$ & 200 \\
\hline
\end{tabular}

Table 2. Cytotoxicity determined by the NCI (USA)

\begin{tabular}{lccc}
\hline compound & $\mathrm{IC}_{50} / \mu \mathrm{M}$ & compound & $\mathrm{IC}_{50} / \mu \mathrm{M}$ \\
\hline $\mathbf{4}$ & 20 & $\mathbf{7 a}$ & $>100$ \\
\hline
\end{tabular}

\section{Conclusions}

In this paper we report the synthesis and cytotoxic evaluation of new annonaceous acetogenine analogues containing a furan ring instead of the naturally occuring tetrahydrofuran ring. The cytotoxic acitivity of the resulting compounds is only in $\mu \mathrm{M}$ range and so much than that the natural products with a cytotoxicity in $\mathrm{nM}$ range.

In summary, we describe here a shorter and more efficient way towards the annoceous acetogenine skeleton. The resulting compounds might be an interesting starting material for the synthesis of enantiomeric pure tetrahydrofuran analogues.

\section{Experimental}

IR-Spectra: Jasco FT-IR Paragon; MS: Hewlett Packard MS-Engine, electron ionisation (EI) $70 \mathrm{eV}$, chemical ionisation (CI) with $\mathrm{CH}_{4}(300 \mathrm{eV})$; NMR (400 MHz): Jeol GSX $400\left({ }^{1} \mathrm{H}: 400 \mathrm{MHz},{ }^{13} \mathrm{C}: 100 \mathrm{MHz}\right)$; GLCMS: Shimadzu GC 17 A; flash column chromatography (FCC): silica gel 60 (230-400 mesh, E. Merck, Darmstadt).

\section{5-(11-Hydroxy-undec-1-ynyl)-furan-2-carbaldehyde (3)}

$2.2 \mathrm{~g}$ (10.0 mmol) 5-iodofuran-2-carbaldehyde, 1.7 $\mathrm{g}(10.0 \mathrm{mmol})$ undec-10-yn-1-ol, $200 \mathrm{mg} \mathrm{CuI}$ and 800 $\mathrm{mg} \mathrm{PdCl}\left(\mathrm{PPh}_{3}\right)_{2}$ were dissolved in $20 \mathrm{ml}$ EDMA and stirred for $12 \mathrm{~h}$ at room temperature. The solvent was evaporated and the residue was dissolved in $20 \mathrm{~mL} 5 \%$ aqueous $\mathrm{Na}_{2} \mathrm{~S}_{2} \mathrm{O}_{3}$ solution. The solution was extracted with diethyl ether $(3 \times 25 \mathrm{~mL})$. The combined organic layers were dried over $\mathrm{Na}_{2} \mathrm{SO}_{4}$, the solvent was evaporated and the residue was purified by FCC ( $n$-hexane/ethyl acetat 5:1) to give $1.8 \mathrm{~g}(69 \%)$ of $\mathbf{3}$ as a brown solid. IR $(\mathrm{KBr}$ disc $) v_{\max } / \mathrm{cm}^{-1}: 3405,3337,3101$, 2920, 2835, 2223, 1677, 1510, 1271, 819, 763. EI-MS $\mathrm{m} / \mathrm{z}$ (rel. int.): $262\left(\mathrm{M}^{+}, 20\right), 190$ (25), 136 (100). HRMS: Calc.: 262.1569. Found: 262.1578. ${ }^{1} \mathrm{H}$ NMR (400 $\left.\mathrm{MHz}, \mathrm{CDCl}_{3}\right) \delta(\mathrm{J}, \mathrm{Hz}) 1.38\left(\mathrm{~m}, 10 \mathrm{H}, 5 \mathrm{CH}_{2}\right), 1.59(\mathrm{~m}$, $\left.4 \mathrm{H}, 2 \mathrm{CH}_{2}\right), 2.46\left(\mathrm{t}, J 7.6,2 \mathrm{H}, \mathrm{CH}_{2}\right), 3.65$ (t, $J$ 7.2, 2 $\left.\mathrm{H}, \mathrm{CH}_{2} \mathrm{O}\right), 6.60(\mathrm{~d}, J 3.6,1 \mathrm{H}$, aromat. $\mathrm{CH}), 7.20(\mathrm{~d}, J$ 3.6, $1 \mathrm{H}$, aromat. $\mathrm{CH}), 9.58(\mathrm{~s}, 1 \mathrm{H}, \mathrm{CHO}) .{ }^{13} \mathrm{C}$ NMR $\left(400 \mathrm{MHz}, \mathrm{CDCl}_{3}\right) \delta 21.1\left(\mathrm{CH}_{2}\right), 25.7\left(\mathrm{CH}_{2}\right), 28.0$ $\left(\mathrm{CH}_{2}\right), 28.8\left(\mathrm{CH}_{2}\right), 29.0\left(\mathrm{CH}_{2}\right), 29.3\left(\mathrm{CH}_{2}\right), 29.4\left(\mathrm{CH}_{2}\right)$, $32.8\left(\mathrm{CH}_{2}\right), 63.0\left(\mathrm{CH}_{2}\right), 70.4$ (quat. C), 99.0 (quat. C), 115.9 (2 aromat. $\mathrm{CH}$ ), 142.6 (quat. $\mathrm{C}$ ), 151.9 (quat. C), 177.1 (CHO).

\section{1-[5-(11-Hydroxy-undec-1-ynyl)-furan-2-yl]tridecan-1-ol} (4)

$1.7 \mathrm{~g}(6.5 \mathrm{mmol})$ of $\mathbf{3}$ were dissolved in $10 \mathrm{~mL}$ dry THF and $13 \mathrm{~mL}$ of $1 \mathrm{~mol} \mathrm{~L}^{-1}(13 \mathrm{mmol})$ dodecylmagnesium bromide solution in $n$-hexane were added dropwise. The mixture was stirred for $10 \mathrm{~h}$, was diluted with $20 \mathrm{~mL}$ saturated $\mathrm{NH}_{4} \mathrm{Cl}$ solution and was extracted with diethyl ether $(3 \times 30 \mathrm{~mL})$. The combined organic layers were dried over $\mathrm{Na}_{2} \mathrm{SO}_{4}$, the solvent was evaporated and the residue was purified by FCC ( $n$-hexane/ethyl acetate 5:1) to give $1.34 \mathrm{~g}(48 \%)$ of 4 as a brown oil. CI-MS: $\mathrm{m} / \mathrm{z}$ (rel. int.): $460\left(\mathrm{M}^{+}, 6\right), 443$ (100), 304 (34), 259 (38). HR-MS: Calc.: 460.3553 Found: 460.3538. ${ }^{1} \mathrm{H}$ NMR (400 MHz, $\left.\mathrm{CDCl}_{3}\right)$ $\delta(J, \mathrm{~Hz}) 0.88\left(\mathrm{t}, J 6.7,3 \mathrm{H}, \mathrm{CH}_{3}\right), 1.28\left(\mathrm{~m}, 20 \mathrm{H}, 10 \mathrm{CH}_{2}\right)$, $1.43\left(\mathrm{~m}, 4 \mathrm{H}, 2 \mathrm{CH}_{2}\right), 1.59\left(\mathrm{~m}, 6 \mathrm{H}, 3 \mathrm{CH}_{2}\right), 1.82(\mathrm{~m}, 2 \mathrm{H}$, $\mathrm{CH}_{2}$ ), 2.43 (t, $J$ 7.7, $\left.2 \mathrm{H}, \mathrm{CH}_{2}\right), 3.64\left(\mathrm{t}, J 6.4,2 \mathrm{H}, \mathrm{CH}_{2} \mathrm{O}\right.$ ), $4.62(\mathrm{t}, J 6.4,1 \mathrm{H}, \mathrm{CH}), 6.18(\mathrm{~d}, J 3.2,1 \mathrm{H}$, aromat. $\mathrm{CH})$, $6.40(\mathrm{~d}, J 3.2,1 \mathrm{H}$, aromat. $\mathrm{CH}) .{ }^{13} \mathrm{C}$ NMR $(400 \mathrm{MHz}$, $\left.\mathrm{CDCl}_{3}\right): \delta 14.2\left(\mathrm{CH}_{3}\right), 22.8\left(\mathrm{CH}_{2}\right), 25.6\left(\mathrm{CH}_{2}\right), 25.7\left(\mathrm{CH}_{2}\right)$, $25.8\left(\mathrm{CH}_{2}\right), 28.4\left(\mathrm{CH}_{2}\right), 28.8\left(\mathrm{CH}_{2}\right), 29.0\left(\mathrm{CH}_{2}\right), 29.3\left(\mathrm{CH}_{2}\right)$, $29.4\left(\mathrm{CH}_{2}\right), 29.4\left(\mathrm{CH}_{2}\right), 29.5\left(\mathrm{CH}_{2}\right), 29.6\left(\mathrm{CH}_{2}\right), 29.6\left(\mathrm{CH}_{2}\right)$, $29.7\left(\mathrm{CH}_{2}\right), 29.8\left(\mathrm{CH}_{2}\right), 32.0\left(\mathrm{CH}_{2}\right), 32.8\left(\mathrm{CH}_{2}\right), 35.6\left(\mathrm{CH}_{2}\right)$, $63.1\left(\mathrm{CH}_{2} \mathrm{O}\right.$ ), $67.9(\mathrm{CH}), 71.0$ (quat. C), 94.9 (quat. C), 106.8 (aromat. $\mathrm{CH}$ ), 114.4 (aromat. $\mathrm{CH}$ ), 136.8 (quat. C), 157.2 (quat. C).

11-Hydroxy-1-[5-(1-hydroxy-tridecyl)-furan-2-yl]undecan-1-one (5)

$200 \mathrm{mg}(0.4 \mathrm{mmol})$ of 4 were dissolved in $20 \mathrm{~mL}$ methanol, $10 \mathrm{~mL} \mathrm{5 \%}$ aqueous $\mathrm{H}_{2} \mathrm{SO}_{4}$ and $200 \mathrm{mg}(0.9$ mmol) yellow $\mathrm{HgO}$ were added. The solution was stirred for $12 \mathrm{~h}$ at room temperature. The methanol was evaporated and residue was quenched with $20 \mathrm{~mL}$ saturated $\mathrm{Na}_{2} \mathrm{CO}_{3}$ solution and extracted with diethyl ether $(3 \times 30 \mathrm{~mL})$. The combined organic layers were dried over $\mathrm{Na}_{2} \mathrm{SO}_{4}$ and the residue was purified by FCC ( $n$-hexane/ethyl acetate $5: 1$ ) to give $50 \mathrm{mg}(28 \%)$ of $\mathbf{5}$ 
as a brown oil. HR-MS: Calc.: 450.3709. Found: 450.3708. ${ }^{1} \mathrm{H}$ NMR $\left(400 \mathrm{MHz}, \mathrm{CDCl}_{3}\right) \delta(J, \mathrm{~Hz}) 0.88$ $\left(\mathrm{t}, J 6.8,3 \mathrm{H}, \mathrm{CH}_{3}\right), 1.29\left(\mathrm{~m}, 28 \mathrm{H}, 14 \mathrm{CH}_{2}\right), 1.56(\mathrm{~m}, 4$ $\left.\mathrm{H}, 2 \mathrm{CH}_{2}\right), 1.71\left(\mathrm{~m}, 4 \mathrm{H}, 2 \mathrm{CH}_{2}\right), 1.86\left(\mathrm{~m}, 2 \mathrm{H}, \mathrm{CH}_{2}\right)$, $2.78\left(\mathrm{t}, J 8.8,2 \mathrm{H}, \mathrm{CH}_{2}\right), 3.64\left(\mathrm{t}, J 6.6,2 \mathrm{H}, \mathrm{CH}_{2} \mathrm{O}\right)$, $4.74(\mathrm{t}, J 6.5,1 \mathrm{H}, \mathrm{CH}), 6.38(\mathrm{dd}, J 0.6, J 3.6,1 \mathrm{H}$, aromat. $\mathrm{CH}), 7.13(\mathrm{~d}, J 3.6,1 \mathrm{H}$, aromat. $\mathrm{CH}) .{ }^{13} \mathrm{C} \mathrm{NMR}$ $\left(400 \mathrm{MHz}, \mathrm{CDCl}_{3}\right): \delta 14.1\left(\mathrm{CH}_{3}\right), 22.7\left(\mathrm{CH}_{2}\right), 24.5$ $\left(\mathrm{CH}_{2}\right), 25.3\left(\mathrm{CH}_{2}\right), 25.7\left(\mathrm{CH}_{2}\right), 29.2\left(\mathrm{CH}_{2}\right), 29.3(3 \mathrm{x}$ $\left.\mathrm{CH}_{2}\right), 29.4\left(2 \times \mathrm{CH}_{2}\right), 29.5\left(\mathrm{CH}_{2}\right), 29.5\left(\mathrm{CH}_{2}\right), 29.6$ $\left(\mathrm{CH}_{2}\right), 29.7\left(\mathrm{CH}_{2}\right), 29.8\left(\mathrm{CH}_{2}\right), 31.9\left(\mathrm{CH}_{2}\right), 32.7\left(\mathrm{CH}_{2}\right)$, $35.7\left(\mathrm{CH}_{2}\right), 38.4\left(\mathrm{CH}_{2}\right), 63.1\left(\mathrm{CH}_{2} \mathrm{O}\right), 68.0(\mathrm{CH}), 108.0$ (aromat. $\mathrm{CH}$ ), 118.1 (aromat. $\mathrm{CH}$ ), 151.9 (quat. C), 161.7 (quat. C), 189.7 (CO).

\section{1-(5-Formyl-furan-2-yl)-undec-10-ynoic acid methyl ester $(\boldsymbol{6 a})$}

The compound was prepared as described for $\mathbf{3}$ from $1.9 \mathrm{~g}(10 \mathrm{mmol})$ methyl undecynoate and $2.2 \mathrm{~g}(10$ mmol) 5-iodofuran carbaldehyde to give $2.6 \mathrm{~g}(90 \%)$ of $\mathbf{6 a}$ as a brown solid. CI-MS $m / z$ (rel.int.): $291\left(\mathbf{M}^{+}+1\right.$, 34), 259 (100). HR-MS: Calc.: 290.1518. Found: 290.1553. ${ }^{1} \mathrm{H}$ NMR (400 MHz, $\left.\mathrm{CDCl}_{3}\right): \delta(J, \mathrm{~Hz}) 1.29$ $\left(\mathrm{m}, 6 \mathrm{H}, 3 \mathrm{CH}_{2}\right), 1.61\left(\mathrm{~m}, 4 \mathrm{H}, 2 \mathrm{CH}_{2}\right), 2.31(\mathrm{t}, J$ 7.0, 2 $\left.\mathrm{H}, \mathrm{CH}_{2}\right), 2.45\left(\mathrm{t}, J 7.0,2 \mathrm{H}, \mathrm{CH}_{2}\right), 3.67\left(\mathrm{~s}, 1 \mathrm{H}, \mathrm{OCH}_{3}\right)$, $6.60(\mathrm{~d}, J 4.0,1 \mathrm{H}$, aromat. $\mathrm{CH}), 7.20(\mathrm{dd}, J 4.0, J 0.4$, $1 \mathrm{H}$, aromat. $\mathrm{CH}), 9.58(\mathrm{~d}, J 0.4,1 \mathrm{H}, \mathrm{CHO}) .{ }^{13} \mathrm{C} \mathrm{NMR}$ $\left(400 \mathrm{MHz}, \mathrm{CDCl}_{3}\right): \delta 19.5,22.70\left(\mathrm{CH}_{2}\right), 24.9\left(\mathrm{CH}_{2}\right)$, $28.0\left(\mathrm{CH}_{2}\right), 28.8\left(\mathrm{CH}_{2}\right), 28.9\left(\mathrm{CH}_{2}\right), 29.1\left(\mathrm{CH}_{2}\right), 34.1$ $\left(\mathrm{CH}_{2}\right), 51.5\left(\mathrm{OCH}_{3}\right), 70.4$ (quat. C), 98.9 (quat. C), 115.9 (2 aromat. $\mathrm{CH}$ ), 142.6 (quat. C), 151.9 (quat. $\mathrm{C}$ ), $174.4(\mathrm{CO})$.

\section{1-(5-Formyl-furan-2-yl)-undec-10-ynoic acid 1-ethoy- carbonyl ethyl ester (6b)}

The compound was prepared as described for $\mathbf{3}$ from $0.5 \mathrm{~g}(1.8 \mathrm{mmol}) \mathbf{2 b}$ and $0.4 \mathrm{~g}(1.8 \mathrm{mmol}) 5$-iodofuran carbaldehyde to give $610 \mathrm{mg}(90 \%)$ of $\mathbf{6 b}$ as a brown solid. CI-MS $m / z$ (rel. int.): $377\left(\mathrm{M}^{+}+1,12\right), 259$ (100). HR-MS: Calc.: 376.1886 . Found: $376.1875 .{ }^{1} \mathrm{H}$ NMR $\left(400 \mathrm{MHz}, \mathrm{CDCl}_{3}\right): \delta(J, \mathrm{~Hz}) 1.27\left(\mathrm{t}, J 7.5,3 \mathrm{H}, \mathrm{CH}_{3}\right)$, $1.34\left(\mathrm{~m}, 6 \mathrm{H}, 3 \mathrm{CH}_{2}\right), 1.43\left(\mathrm{~m}, 2 \mathrm{H}, \mathrm{CH}_{2}\right), 1.48(\mathrm{~d}, J$ 7.2, $\left.3 \mathrm{H}, \mathrm{CH}_{3}\right), 1.61\left(\mathrm{~m}, 4 \mathrm{H}, 2 \mathrm{CH}_{2}\right), 2.38\left(\mathrm{~m}, 2 \mathrm{H}, \mathrm{CH}_{2}\right)$, $2.45\left(\mathrm{t}, J 6.6,2 \mathrm{H}, \mathrm{CH}_{2}\right), 4.20\left(\mathrm{q}, J 7.5,2 \mathrm{H}, \mathrm{CH}_{2} \mathrm{O}\right)$, $5.06(\mathrm{q}, J 7.2,1 \mathrm{H}, \mathrm{CH}), 6.60(\mathrm{~d}, J 3.9,1 \mathrm{H}$, aromat. $\mathrm{CH}), 7.20(\mathrm{~d}, J 3.9,1 \mathrm{H}$, aromat. $\mathrm{CH}), 9.58(\mathrm{~s}, 1 \mathrm{H}$, CHO). ${ }^{13} \mathrm{C}$ NMR (400 MHz, $\left.\mathrm{CDCl}_{3}\right): \delta 14.0\left(\mathrm{CH}_{3}\right), 16.9$ $\left(\mathrm{CH}_{3}\right), 19.4\left(\mathrm{CH}_{2}\right), 24.7\left(\mathrm{CH}_{2}\right), 27.9\left(\mathrm{CH}_{2}\right), 28.7\left(\mathrm{CH}_{2}\right)$, $28.8\left(\mathrm{CH}_{2}\right), 28.9\left(\mathrm{CH}_{2}\right), 29.0\left(\mathrm{CH}_{2}\right), 33.9\left(\mathrm{CH}_{2}\right), 61.2$
$\left(\mathrm{CH}_{2} \mathrm{O}\right), 68.4(\mathrm{CH}), 70.3$ (quat. C), 98.8 (quat. C), 142.5 (quat. C), 151.8 (quat. C), 115.8 (2 aromat. $\mathrm{CH}$ ), 170.9 (CO), 173.1 (CO), 177.0 (CHO).

11-[5-(1-Hydroxy-tridecyl)-furan-2-yl]-undec-10-ynoic acid methyl ester (7a)

The compound was prepared as described for $\mathbf{4}$ from $200 \mathrm{mg}(0.7 \mathrm{mmol})$ of $\mathbf{6 a}$ and $2 \mathrm{~mL} 1 \mathrm{~mol} \mathrm{~L}^{-1}(2.0 \mathrm{mmol})$ dodecyl magnesium bromide solution in $\mathrm{n}$-hexane to give $200 \mathrm{mg}(62 \%)$ of $\mathbf{7 a}$ as a yellow oil. CI-MS $\mathrm{m} / \mathrm{z}$ (rel. int.): $460\left(\mathrm{M}^{+}, 6\right), 443$ (100), 304 (34). HR-MS: Calc.: 460.3553 Found: 460.3538. ${ }^{1} \mathrm{H}$ NMR $\left(400 \mathrm{MHz}, \mathrm{CDCl}_{3}\right): \delta(\mathrm{J}, \mathrm{Hz})$ $0.81\left(\mathrm{t}, J\right.$ 7 .0, $\left.3 \mathrm{H}, \mathrm{CH}_{3}\right), 1.19\left(\mathrm{~m}, 20 \mathrm{H}, 10 \mathrm{CH}_{2}\right), 1.33(\mathrm{~m}$, $\left.6 \mathrm{H}, 3 \mathrm{CH}_{2}\right), 1.53\left(\mathrm{~m}, 6 \mathrm{H}, 3 \mathrm{CH}_{2}\right), 1.75\left(\mathrm{~m}, 2 \mathrm{H}, \mathrm{CH}_{2}\right)$, $2.23\left(\mathrm{t}, J 8.7,2 \mathrm{H}, \mathrm{CH}_{2}\right), 2.35\left(\mathrm{t}, J 7.7,2 \mathrm{H}, \mathrm{CH}_{2}\right), 3.59$ (s, $\left.3 \mathrm{H}, \mathrm{OCH}_{3}\right), 4.54(\mathrm{t}, J 6.7,1 \mathrm{H}, \mathrm{CH}), 6.11(\mathrm{~d}, J 3.0,1 \mathrm{H}$, aromat. $\mathrm{CH}), 6.33(\mathrm{~d}, J 3.0,1 \mathrm{H}$, aromat. $\mathrm{CH}) .{ }^{13} \mathrm{C} \mathrm{NMR}$ $\left(400 \mathrm{MHz}, \mathrm{CDCl}_{3}\right): \delta 14.09\left(\mathrm{CH}_{3}\right), 22.7-35.5\left(19 \mathrm{CH}_{2}\right)$, $51.4(\mathrm{CH}), 67.9\left(\mathrm{OCH}_{3}\right), 74.5$ (quat. C), 94.8 (quat. C), 106.7 (aromat. $\mathrm{CH}$ ), 114.3 (aromat. $\mathrm{CH}$ ), 136.8 (quat. C), 157.3 (quat. C), $174.3(\mathrm{CO})$.

11-[5-(1-Hydroxy-tridecyl)-furan-2-yl]-undec-10-ynoic acid 1-ethoxycarbonyl-ethyl ester (7a)

The compound was prepared as described for $\mathbf{4}$ from $263 \mathrm{mg}(0.7 \mathrm{mmol})$ of $\mathbf{6 b}$ and $2 \mathrm{~mL} 1 \mathrm{~mol} \mathrm{~L}^{-1}(2.0 \mathrm{mmol})$ dodecyl magnesium bromide solution in $n$-hexane to give $300 \mathrm{mg}(78 \%)$ of $\mathbf{7 b}$ as a yellow oil. HR-MS: Calc.: 546.3920. Found: 546.3915. CI-MS $\mathrm{m} / \mathrm{z}$ (rel. int.): 546 $\left(\mathrm{M}^{+}, 6\right), 529$ (100), 304 (54), 259 (64). ${ }^{1} \mathrm{H}$ NMR (400 MHz, $\left.\mathrm{CDCl}_{3}\right): \delta(J, \mathrm{~Hz}) 0.88\left(\mathrm{t}, J\right.$ 7.1, $\left.3 \mathrm{H}, \mathrm{CH}_{3}\right), 0.88(\mathrm{t}, J$ 7.1, $\left.3 \mathrm{H}, \mathrm{CH}_{3}\right), 1.27\left(\mathrm{~m}, 24 \mathrm{H}, 12 \mathrm{CH}_{2}\right), 1.49(\mathrm{~d}, J 6.8,3 \mathrm{H}$, $\left.\mathrm{CH}_{3}\right), 1.61\left(\mathrm{~m}, 4 \mathrm{H}, 2 \mathrm{CH}_{2}\right), 1.82\left(\mathrm{~m}, 4 \mathrm{H}, 2 \mathrm{CH}_{2}\right), 1.99$ $\left(\mathrm{m}, 2 \mathrm{H}, \mathrm{CH}_{2}\right), 2.41\left(\mathrm{~m}, 4 \mathrm{H}, 2 \mathrm{CH}_{2}\right), 4.20(\mathrm{q}, J 7.1,2 \mathrm{H}$, $\left.\mathrm{CH}_{2}\right), 4.62(\mathrm{~m}, 1 \mathrm{H}, \mathrm{CH}), 5.07$ (q, J 6.8, $\left.1 \mathrm{H}, \mathrm{CH}\right), 6.19$ (d, $J 3.4,1 \mathrm{H}$, aromat. $\mathrm{CH}), 6.41(\mathrm{~d}, J 3.4,1 \mathrm{H}$, aromat. $\mathrm{CH}) .{ }^{13} \mathrm{C}$ NMR $\left(400 \mathrm{MHz}, \mathrm{CDCl}_{3}\right): \delta 14.1\left(\mathrm{CH}_{3}\right), 14.1$ $\left(\mathrm{CH}_{3}\right), 16.9\left(\mathrm{CH}_{3}\right), 19.5\left(\mathrm{CH}_{2}\right), 22.7\left(\mathrm{CH}_{2}\right), 24.8\left(\mathrm{CH}_{2}\right)$, $25.5\left(\mathrm{CH}_{2}\right), 28.3\left(\mathrm{CH}_{2}\right), 28.8\left(\mathrm{CH}_{2}\right), 28.9\left(\mathrm{CH}_{2}\right), 29.0$ $\left(\mathrm{CH}_{2}\right), 29.1\left(\mathrm{CH}_{2}\right), 29.4\left(\mathrm{CH}_{2}\right), 29.5\left(\mathrm{CH}_{2}\right), 29.6\left(\mathrm{CH}_{2}\right)$, $29.7\left(3 \mathrm{CH}_{2}\right), 29.7\left(\mathrm{CH}_{2}\right), 31.9\left(\mathrm{CH}_{2}\right), 34.0\left(\mathrm{CH}_{2}\right), 35.5$ $\left(\mathrm{CH}_{2}\right), 61.3\left(\mathrm{CH}_{2}\right), 68.5(\mathrm{CH}), 67.9(\mathrm{CH}), 71.0$ (quat. C), 94.9 (quat. C), 106.7 (aromat. $\mathrm{CH}$ ), 114.4 (aromat. $\mathrm{CH}$ ), 136.8 (quat. C), 157.2 (quat. C), 171.0 (CO), 173.2 (CO).

\section{Acknowledgments}

We are greatly indebted to Tanja Höft and Martina Stadler for technical support. 


\section{References}

1. Hoppe, R.; Scharf, H.-D.; Synthesis 1995, 1447.

2. Liaw, Ch.-Ch.; Chang, F.-R.; Lin, Ch.-J. Chou, Ch.-Y.; Chiu, H.-F.; Wu, M.-J.; Wu, Y.-Ch.; J. Nat. Prod. 2002, 65, 470.

3. Krauss, J.; Unterreitmeier, D.; Arch. Pharm. Pharm. Med. 2003, 336, 381.

4. Hennion, G. F.; Pillar, C. J.; J. Am Chem. Soc. 1950, 72, 5317.
5. Mosmann, T. J.; Immunol. Methods 1983, 65, 55.

6. http://dtp.nci.nih.gov/docs/misc/common_files/ submit_compounds.html (acessed in 2006/2007).

7. Emde, U.; Koert, U.; Tetrahedron Lett. 1999, 40, 5979.

8. Prestat, G.; Baylon, C.; Heck, M.; Grasa, G.; Nolan, S.; Mioskowski, C.; J. Org. Chem. 2004, 69, 5770.

Received: August 9, 2006 Web Release Date: April 30, 2007 